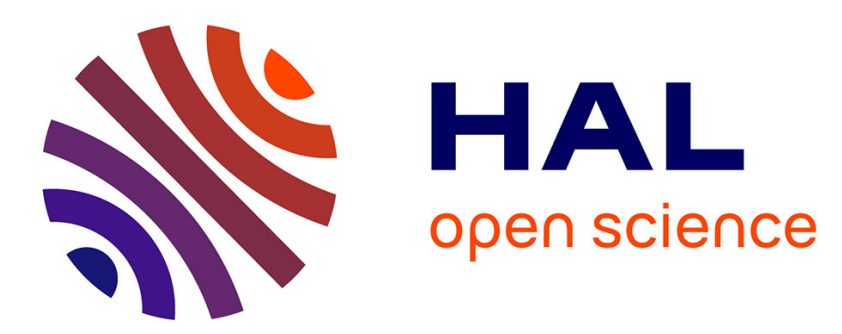

\title{
Novel theoretical aspects on photorefractive ultrasonic detection and implementation of a sensor with an optimum sensitivity
}

Louis-Anne de Montmorillon, Philippe Delaye, Jean-Claude Launay, Gérald Roosen

\section{To cite this version:}

Louis-Anne de Montmorillon, Philippe Delaye, Jean-Claude Launay, Gérald Roosen. Novel theoretical aspects on photorefractive ultrasonic detection and implementation of a sensor with an optimum sensitivity. Journal of Applied Physics, 1997, 82 (12), pp.5913-5922. 10.1063/1.366492 . hal-00673502

HAL Id: hal-00673502

https://hal-iogs.archives-ouvertes.fr/hal-00673502

Submitted on 23 Feb 2012

HAL is a multi-disciplinary open access archive for the deposit and dissemination of scientific research documents, whether they are published or not. The documents may come from teaching and research institutions in France or abroad, or from public or private research centers.
L'archive ouverte pluridisciplinaire HAL, est destinée au dépôt et à la diffusion de documents scientifiques de niveau recherche, publiés ou non, émanant des établissements d'enseignement et de recherche français ou étrangers, des laboratoires publics ou privés. 


\title{
Novel theoretical aspects on photorefractive ultrasonic detection and implementation of a sensor with an optimum sensitivity
}

\author{
Louis-Anne de Montmorillon and Philippe Delaye ${ }^{\mathrm{a})}$ \\ Institut d'Optique, Unité de Recherche Associée 14 au Centre National de la Recherche Scientifique, \\ Bâtiment 503, Centre Scientifique d'Orsay, B.P. 147, 91403 Orsay Cedex, France \\ Jean-Claude Launay \\ 3-AR-Action Aquitaine de Recherche en Apesanteur, B.P. 11, 33165 Saint Médard en Jalles Cedex, France \\ and ICMCB-Institut de Chimie de la Matière Condensée de Bordeaux, Avenue du Dr. A. Schweitzer, \\ 33608 Pessac Cedex, France \\ Gérald Roosen \\ Institut d'Optique, Unité de Recherche Associée 14 au Centre National de la Recherche Scientifique, \\ Bâtiment 503, Centre Scientifique d'Orsay, B.P. 147, 91403 Orsay Cedex, France
}

(Received 10 June 1997; accepted for publication 9 September 1997)

\begin{abstract}
We here expose theoretical and experimental results on homodyne detection using near-infrared laser sources, at $1.06,1.32$, and $1.55 \mu \mathrm{m}$ wavelengths. The used photorefractive crystals are two large size CdZnTe:V samples. With speckled beams such as the ones scattered by diffusive objects, we reach a detection limit which, at $1.55 \mu \mathrm{m}$, is only 1.6 times above the one obtained with plane waves in a classical interferometer and only 2 and 2.2 times above at 1.32 and $1.06 \mu \mathrm{m}$, respectively. It is then demonstrated that the electron-hole competition, which varies enormously between these three wavelengths and gives a nearly zero two-wave-mixing gain at $1.32 \mu \mathrm{m}$, does not influence the sensitivity of the system. Moreover, we show that the frequency cutoff of the system is four times higher in the attenuation regime than in the amplification one. (c) 1997 American Institute of Physics. [S0021-8979(97)00824-4]
\end{abstract}

\section{INTRODUCTION}

Laser ultrasonics is a technique now widely used in the field of nondestructive testing. ${ }^{1,2}$ Its main advantage, over classical piezoelectric techniques, is its noncontact and even remote character. This point is of importance for a development of such systems in industry when inspection of hot materials or of products moving on a production line is considered. The ultrasound wave is detected through the small surface displacement (few tenths of nanometers, with a frequency ranging, typically, from some hundreds of $\mathrm{kHz}$ to some hundreds of $\mathrm{MHz}$ ), it induces when reflecting at the surface of the tested sample. A laser incident on this surface is, thus, phase modulated with a phase modulation directly proportional to the surface displacement. This phase modulation is then detected by a coherent detection system. Here lies the main problem of the optical detection of ultrasounds. Most of these coherent detection systems have indeed a sensitivity to the surface displacement that is greatly reduced as soon as the tested surface is no longer a polished surface. This reduction of sensitivity is incompatible with the use of laser ultrasonics in the industry, as it accentuates the major drawbacks of optical detection of ultrasounds, i.e., its lower sensitivity compared to piezoelectric transduction. ${ }^{3}$ Thus, to be considered, an optical system will have to be as close as possible to the optimum performances, whatever the surface roughness.

One solution proposed for the detection of phase modulation on the speckled beam issued from a scattering surface was the confocal Fabry-Perot interferometer. ${ }^{2,4,5}$ The performances of this interferometer were sufficient to permit the

${ }^{\text {a)} E l e c t r o n i c ~ m a i l: ~ p h i l i p p e . d e l a y e ~ @ i o t a . u-p s u d ~}$ industrial development of a laser ultrasonics system. ${ }^{6}$ Nevertheless, the confocal Fabry-Perot interferometer suffers from a reduced sensitivity when regarding the detection of lowfrequency (below $2 \mathrm{MHz}$ ) ultrasonic waves, which limits its use. This was one of the reasons for considering active or adaptive holographic interferometers. Among different possibilities, double phase conjugation, ${ }^{7-9}$ non-steady-state photoelectromotive force, ${ }^{10}$ and photorefractive beam combiner, ${ }^{11-16}$ we analyzed and developed this later technique that seems to us as the most promising.

The operating principle of this photorefractive beam combiner based on two-wave mixing is the following (Fig. 1): Two beams, one signal beam issued from the tested scattering surface and one pump beam directly issued from the same laser and coherent with the signal beam, are sent on the photorefractive crystal. The signal beam is a speckled phase modulated beam. The two beams write in the photorefractive crystal a hologram of the signal beam wave-front structure. This hologram is a stationary hologram, as the phase modulation caused by the surface displacement is generally of the order of some nanometers at maximum. The pump beam diffracts on this hologram, and thus, creates in the direction of the transmitted signal beam a local oscillator with exactly the same wave-front structure. This local oscillator beam then interferes with the signal beam in a homodyne detection scheme, allowing a perfect detection of the phase modulation, whatever the spatial structure of the signal beam. This gives a high field of view homodyne detection. The principle works at high frequencies of the phase modulation, i.e., at frequencies higher than the inverse of the material response time. When the signal varies on periods of the order or larger than the response time of the photorefractive effect, the hologram follows the phase modulation and this phase modu- 


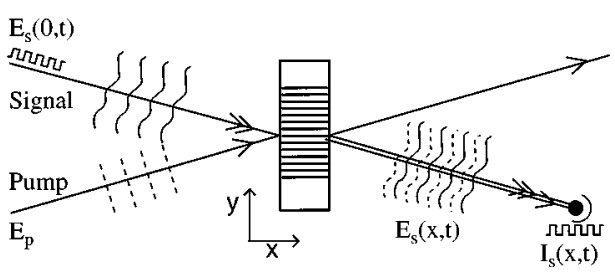

FIG. 1. Schematic representation of the two-wave-mixing photorefractive technique to detect vibrations.

lation is transmitted to the local oscillator, strongly decreasing the demodulation efficiency of the system. The system, thus, possesses a high pass frequency response. Its cutoff frequency is defined by the photorefractive properties and can be adapted by the correct choice of pump beam power. The high-frequency limit is fixed by the used detector and its electronics. The high-frequency ultrasound signal is, thus, perfectly detected, whereas low-frequency vibrations (dc to $10 \mathrm{kHz}$ ), that obviously exist in an industrial environment and generally perturb the measurement, are eliminated. We see that we meet all the requirements of a laser ultrasonic detection system, i.e., a large frequency bandwidth and the possibility of working with a speckled beam. The photorefractive beam combiner also adds the advantage of being very simple to implement as the only adjustment consists here to superpose the signal and pump beams in the photorefractive crystal, whereas all passive interferometric techniques need very precise alignments and active stabilizations of the interferometer.

We present in this paper a theoretical and experimental study of this photorefractive sensor. We mainly insist on the optimization of the sensor sensitivity to the detection of small ultrasonic displacements that is the delicate point of optical systems. Among different implementation possibilities, ${ }^{17}$ we choose the simplest one where the crystal is used in the beam coupling geometry with an applied electric field to assure a good phase demodulation..$^{15}$ The photorefractive crystal we use is vanadium doped zinc-cadmium telluride $(\mathrm{CdZnTe}: \mathrm{V})$, and the study is performed at the three wavelengths of $1.06,1.32$, and $1.55 \mu \mathrm{m}$. First, a theoretical modeling of the photorefractive beam combiner is presented in Sec. II. We then show in Sec. III that under a dc electric field, the sensitivity of the system is almost independent of the electron-hole competition in the crystal. In Sec. IV, we present experimental measurements of the detection sensitivity of the sensor. Finally, in Sec. V, the frequency response of the device is studied.

\section{DETECTION OF AN ULTRASONIC PHASE MODULATED SIGNAL}

We first consider that the signal and pump beams are plane waves, but the spirit of the calculation is adapted to speckled beams (as explained later). We consider that there is no depletion of the pump beam, due to energy transfer, i.e., we have a large pump-to-signal irradiance ratio. This, in fact, corresponds to the operating condition of the sensor, as the signal beam intensity scattered off the tested surface is generally weak. With the small photorefractive gain of semi- conductor crystals, a value of 10 for this pump-to-signal ratio is enough. However, we will take into account the attenuation of the beams due to the absorption of the crystal. We define $E_{s}(x, t)$ and $E_{p}(x, t)$, the amplitudes of the emerging signal and pump beams, after a propagation distance $x$ in the crystal and at a time $t$ after the beginning of the experiment (Fig. 1). As the photorefractive response time is inversely proportional to the pump beam irradiance, this response time will increase with $x$. The amplitude of the emerging signal beam has been determined using the Laplace transform formalism, ${ }^{18}$ but is here given in a more useful formulation. The signal expression has been reshaped in order to eliminate the derivative term leading to an expression that appears like a filter equation.

It results, assuming that at $t=0$ there is no grating recorded in the photorefractive material, in

$$
E_{s}(x, t)=e^{-\alpha x / 2}\left[E_{s}(0, t)+\int_{0}^{t} E_{s}(0, T) G(x, t-T) d T\right],
$$

with $G(x, t)=\frac{e^{-t / \tau_{0}}}{\tau_{0}} \frac{\gamma}{\alpha} \frac{e^{\alpha x}-1}{e^{\alpha x}}{ }_{1} F_{1}\left(\frac{\gamma+\alpha}{\alpha}, 2, \frac{e^{\alpha x}-1}{e^{\alpha x}} \frac{t}{\tau_{0}}\right)$,

where, with the notations of Ref. $18,{ }_{1} F_{1}(a, b, z)$ is a confluent hypergeometric function. ${ }^{19} \alpha$ is the crystal absorption; $\gamma$ is the amplitude photorefractive gain; and $\tau_{0}$ is the photorefractive response time at the entrance of the crystal.

Assuming that the steady-state index grating has been already recorded before the beginning of the temporal modulation of the signal beam at $t=0$, we then obtain

$$
\begin{aligned}
E_{s}(x, t)= & e^{-\alpha x / 2}\left\{\left[e^{\gamma x}-1-\int_{0}^{t} G(x, T) d T\right] E_{s}(0,0)\right. \\
& \left.+E_{s}(0, t)+\int_{0}^{t} E_{s}(0, T) G(x, t-T) d T\right\} .
\end{aligned}
$$

Note that for a nonmodulated signal beam, $E_{s}(0, t)$ $=E_{s}(0,0)$, the grating writing process will be finished at a time $t$, which is large compared to the photorefractive response time. Using expression (1), we obtain

$$
\begin{aligned}
\lim _{t \rightarrow \infty} E_{s}(x, t) & =\lim _{t \rightarrow \infty}\left(e^{-\alpha x / 2} E_{s}(0,0)\left[1+\int_{0}^{t} G(x, T) d T\right]\right) \\
& =e^{-\alpha x / 2} e^{\gamma x} E_{s}(0,0) .
\end{aligned}
$$

We then deduce that

$$
\int_{0}^{\infty} G(x, T) d T=e^{\gamma x}-1,
$$

which allows us to link together the integral of function $G$ and a simple physical parameter: the amplitude photorefractive amplification $e^{\gamma x}$. We see that expressions (1) and (3) are equivalent at time $t$ large compared to the photorefractive response time. This demonstrates an intuitive result: the index grating written in the crystal at a time $t \gg \tau_{0}$ does not depend on the one at time $t=0$.

We can generalize our analytical model to speckled waves. Indeed, a speckled beam can be considered as a su- 
perposition of plane waves and our formalism uses linear equations only. Each of these plane waves will interact with the pump beam identically as spacing variations of written gratings will not significantly affect the photorefractive gain value. However, each of these waves could also mutually interact, which would highly complicate the calculations. Thus, there are in the crystal a "pump-signal" index grating and "signal-signal" index gratings. The effect of these secondary gratings will correspond to an energy transfer from one side of the emerging signal beam cross section to the opposite one, which is not detrimental in our experiment because all the emerging signal beam is collected on a single detector. Moreover, the modulation of the "signal-signal" index gratings will be drastically reduced by the presence of the pump beam as we work with a high pump-to-signal ratio. The "signal-signal" energy transfer can, thus, be considered as negligible. These assumptions were experimentally validated. ${ }^{15}$

We define $E_{s}(0, t)=\bar{E}_{s}+\Delta E_{s}(0, t)$, where $\bar{E}_{s}$ and $\Delta E_{s}(0, t)$ are the respective time averaged and time modulated part of the modulated signal beam. We can now analyze the emerging signal beam, $E_{s}(x, t)$, at a time $t$, which is large compared to the photorefractive response time. As the temporal fluctuations of the signal beam are very rapid compared to the photorefractive response time, i.e., compared to the temporal variations of $G$, we deduce from Eqs. (1) and (5) that

$$
E_{s}(x, t)=e^{-\alpha x / 2}\left[E_{s}(0, t)+\left(e^{\gamma x}-1\right) \bar{E}_{s}\right] .
$$

The component with $\bar{E}_{s}$ corresponds to the diffracted part of the pump beam. It shows that the written index grating is a time averaged hologram. For a small phase modulation of $E_{s}$, we have $\bar{E}_{s}=E_{s}$, but when we increase this modulation, the $\bar{E}_{s}$ value decreases what accounts for the erasing of the hologram. Equation (6) is identical to the results found in Ref. 20, obtained without considering the absorption of the photorefractive material in the dynamics of the two-wave-mixing interaction.

We are experimentally interested by the signal beam intensity, $I_{s}(x, t)=\left|E_{s}(x, t)\right|^{2}$, at the output of the crystal,

$$
\begin{aligned}
I_{s}(x, t)= & \left|E_{s}(x, t)\right|^{2}=e^{-\alpha x}\left\{\left|e^{\gamma x}-1\right|^{2} \bar{E}_{s} \bar{E}_{s}^{*}+\left|E_{s}(0, t)\right|^{2}\right. \\
& \left.+2 \operatorname{Re}\left[\left(e^{\gamma x}-1\right) * \bar{E}_{s}^{*} E_{s}(0, t)\right]\right\} .
\end{aligned}
$$

We consider now the case of the detection of the small amplitude ultrasonic vibrations of a surface. We express the phase modulated signal beam as $E_{s}(0, t)=E_{s} e^{i \varphi(t)} \approx E_{s}(1$ $+i \varphi(t))$. Its averaged part is $\bar{E}_{s} \approx E_{s}$, i.e., we neglect the erasing of the hologram. We write the photorefractive amplitude gain as $\gamma=\gamma^{\prime}+i \gamma^{\prime \prime} .{ }^{18}$ The induced index grating can be considered as the addition of two gratings: one in phase and the other in quadrature with the illumination grating. $\gamma^{\prime}$ represents the part of the gain due to the grating in quadrature and $\gamma^{\prime \prime}$ represents the gain due to the part in phase. Note that the two beam coupling photorefractive gain in energy is $\Gamma=2 \gamma^{\prime}$. The emerging signal beam intensity is, in this case,

$$
I_{s}(x, t)=e^{-\alpha x} I_{s}\left\{e^{2 \gamma^{\prime} x}-2 e^{\gamma^{\prime} x} \sin \left(\gamma^{\prime \prime} x\right) \varphi(t)\right\} .
$$

The modulated part of the detected signal, which is in proportion to the displacements of the tested surface, will be significant when $\gamma^{\prime \prime} \neq 0$ only, i.e., when the index grating and the illumination grating are not $\pi / 2$ phase shifted. This will be the case for a photorefractive semiconductor crystal under a dc external electric field. ${ }^{18}$

We introduce a normalized detection limit, $\delta_{\lim }$, expressed in $\mathrm{nm} \sqrt{\mathrm{W} / \mathrm{Hz}}$. It corresponds to the minimum detectable displacement using a $1 \mathrm{~W}$ signal beam power incident on the crystal and a $1 \mathrm{~Hz}$ detection bandwidth, considering that we operate in the photon noise limited regime (electronic noise is negligible and the laser beam fluctuations are photon noise limited). This allows us to compare different techniques as we will show later. We also define a relative detection limit $\delta_{\text {rel }}$ as the ratio between the detection limit obtained from Eq. (8) for speckled waves and the one obtained with a classical homodyne detection with plane waves, $\delta_{\text {lim }}^{\text {passive }}$. We recall that our aim is to obtain, with the speckled beam, a sensitivity close to the one obtained with a classical interferometer with plane waves. We have

$$
\delta_{\mathrm{lim}}^{\text {passive }}=\frac{\lambda}{4 \pi} \sqrt{\frac{h \nu}{2 \eta}},
$$

where $\lambda$ is the wavelength, $h \nu$ is the photon energy, and $\eta$ is the quantum efficiency of the detector. We, thus, obtain

$$
\delta_{\text {rel }}=\frac{\delta_{\text {lim }}}{\delta_{\text {lim }}^{\text {passive }}}=\frac{e^{\alpha x / 2}}{\left|\sin \left(\gamma^{\prime \prime} x\right)\right|} .
$$

It appears that the relative detection limit does not depend on $\gamma^{\prime}$. It means that we can work in the attenuation $\left(\gamma^{\prime}<0\right)$ or in the amplification $\left(\gamma^{\prime}>0\right)$ regime as well, as long as the detected signal is really photon noise limited. For given $\alpha$ and $\gamma^{\prime \prime}$ values, there is an optimum thickness of the crystal, which allows a minimum value of the relative detection limit. ${ }^{15}$ For a given sample, that is for fixed $\alpha$ and $x$ values, the relative detection limit will be optimized for an applied electric field value giving $\left|\sin \left(\gamma^{\prime \prime} x\right)\right|=1$ (the electricfield value will remain limited to $10 \mathrm{kV} \mathrm{cm}^{-1}$ to avoid any electrical damage). Then, the ultimate sensitivity of the photorefractive sensor will be limited by the losses in the crystal only.

\section{EFFECT OF A DC ELECTRIC FIELD ON THE PHOTOREFRACTIVE GAIN}

In the previous paragraph, we have shown that the detection sensitivity of the sensor depends on the imaginary part, $\gamma^{\prime \prime}$, of the amplitude photorefractive gain. This component has not been deeply studied yet. Indeed, in most applications, the aim is to obtain a maximum $\gamma^{\prime}$ value and a zero $\gamma^{\prime \prime}$ value. In these cases, the $\pi / 2$ phase shift between the index and the illumination gratings is considered as an advantage of the photorefractive effect. ${ }^{21}$ This is realized in the diffusion regime. In these cases, the electron-hole competition $^{22}$ (characterized by an electron-hole competition coefficient, $\left.-1 \leqslant \xi_{0} \leqslant 1\right)$ is unfavorable: when the electron-hole competition is strong $\left(\xi_{0}=0\right)$, the spacecharge field and then the photorefractive gain, $\gamma^{\prime}$, are zero. We will show here that, in the drift regime, $\gamma^{\prime \prime}$ is rather insensitive to electron-hole competition. We begin this study by an intuitive approach. 
We consider here an illumination grating, $I(y)=\bar{I}(1$ $+m \sin k y$ ) (Fig. 1), in a photorefractive crystal, $k$ is the grating wave number and $m$ is the modulation of the pattern. With electron (or hole) dominated conductivity $\left(\left|\xi_{0}\right|=1\right)$, there is in the diffusion regime a local charge grating, which induces a $\pi / 2$ phase shifted space-charge-field grating, $E(y)=\bar{E} \cos (k y)$. Applying a dc electric field on the photorefractive crystal shifts the space-charge-field grating by a phase $\Phi$,

$$
\begin{aligned}
E(y) & =\bar{E} \cos (k y+\Phi) \\
& =\bar{E}(\cos \Phi \cos k y-\sin \Phi \sin k y) .
\end{aligned}
$$

This field can be considered as the sum of a field in phase with $I\left(E_{\text {local }}\right)$ and one in quadrature $\left(E_{\text {quadrature }}\right)$. The photorefractive amplitude gains, $\gamma^{\prime \prime}$ and $\gamma^{\prime}$, are then in proportion with these respective fields:

$$
\gamma^{\prime} \propto \bar{E} \cos \Phi,
$$

and

$$
\gamma^{\prime \prime} \propto-\bar{E} \sin \Phi .
$$

In the diffusion regime, with a perfect electron-hole competition $\left(\xi_{0}=0\right)$, there is no space-charge field. This can be represented as two space-charge fields, $E_{n}$ and $E_{p}$, which have the same amplitude and are $\pi$ phase shifted,

$$
E_{n}(y)=\frac{\bar{E}}{2} \cos (k y),
$$

and

$$
E_{p}(y)=\frac{\bar{E}}{2} \cos (k y+\pi) .
$$

If we apply a dc electric field on the crystal, electron and hole gratings will be $\Phi$ phase shifted in opposite senses

$$
E_{n}(y)=\frac{\bar{E}}{2} \cos (k y+\Phi),
$$

and

$$
E_{p}(y)=\frac{\bar{E}}{2} \cos (k y-\Phi+\pi) .
$$

The total space-charge-field grating in the crystal, which is their sum, is then local

$$
\begin{aligned}
E(y) & =\frac{\bar{E}}{2}[\cos (k y+\Phi)+\cos (k y-\Phi+\pi)] \\
& =-\bar{E} \sin \Phi \sin k y .
\end{aligned}
$$

We, consequently, obtain a zero real amplitude gain and the same imaginary gain as in the case without electron-hole competition,

$$
\gamma^{\prime}=0,
$$

and

$$
\gamma^{\prime \prime} \propto-\bar{E} \sin \Phi .
$$

We generalize this intuitive approach in the case of a variable electron-hole competition factor, $\xi_{0}$. With the same approach as before, we obtain

$$
E_{n}(y)=\left(\frac{1+\xi_{0}}{2}\right) \bar{E} \cos (k y+\Phi),
$$

and

$$
E_{p}(y)=\left(\frac{1-\xi_{0}}{2}\right) \bar{E} \cos (k y-\Phi+\pi),
$$

and so,

$$
\begin{aligned}
E(y) & =E_{n}(y)+E_{p}(y) \\
& =\bar{E}\left(\xi_{0} \cos \Phi \cos k y-\sin \Phi \sin k y\right) .
\end{aligned}
$$

The real and imaginary parts of the amplitude photorefractive gain are then

$$
\gamma^{\prime} \propto \xi_{0} \bar{E} \cos \Phi,
$$

and

$$
\gamma^{\prime \prime} \propto-\bar{E} \sin \Phi .
$$

With this intuitive model, we thus, verify that the real amplitude gain is in proportion with the electron-hole competition factor but we discover, with surprise, that the imaginary gain does not depend on this factor. This result is assessed by a rigorous analysis using a model of the photorefractive effect with one trap center and two types of carriers. The photorefractive amplitude gain is ${ }^{22}$

$$
\gamma=-\frac{\pi n_{0}^{3} r_{\mathrm{eff}}}{\lambda \cos \theta} \frac{k_{B} T}{e} \frac{\left[\frac{\alpha_{n}}{\kappa_{n}^{2}} \frac{k-i V}{k(k-i V) / \kappa_{n}^{2}+1}-\frac{\alpha_{p}}{\kappa_{p}^{2}} \frac{k+i V}{k(k+i V) / \kappa_{p}^{2}+1}\right]}{\left[\frac{A_{n} \alpha_{n}\left(k(k-i V) / k_{0}^{2}+1\right.}{\kappa_{n}^{2}}\left(\frac{A_{p} \alpha_{p}}{k(k-i V) / \kappa_{n}^{2}+1}\right)+\frac{k(k+i V) / k_{0}^{2}+1}{\kappa_{p}^{2}}\left(\frac{k(k+i V) / \kappa_{p}^{2}+1}{k(k)}\right)\right.},
$$

$\alpha_{n(p)}$ are the parts of absorption that generate electrons and holes, respectively; $\kappa_{n(p)}$ are the inverse of the diffusion lengths of electrons and holes, respectively; $k_{0}$ is the inverse of the Debye screening length; $A_{n(p)}=\left(S_{n(p)} I\right.$ $\left.+\beta_{n(p)}\right) / S_{n(p)} I$ with $S_{n(p)}$ the photoionization cross section of electrons (holes), and $\beta_{n(p)}$ the thermal emission coeffi- cient of electrons (holes); $V=e E_{0} / k_{B} T$ with $E_{0}$ the applied electric field. In our experiments, the photoconductivity is much larger than the dark conductivity and we have $A_{n} \approx 1$ and $A_{p} \approx 1$. The electron-hole competition factor $\xi_{0}$ is $\xi_{0}$ $=\left(\alpha_{n}-\alpha_{p}\right) /\left(\alpha_{n}+\alpha_{p}\right)$. Replacing $\alpha_{n}$ and $\alpha_{p}$ by $\alpha_{n}=[(1$ $\left.\left.+\xi_{0}\right) / 2\right] \alpha$ and $\alpha_{p}=\left[\left(1-\xi_{0}\right) / 2\right] \alpha$ in Eq. (26), $\xi_{0}$ becomes 
the only parameter. Note that this factor is experimentally measured in a two-beam coupling experiment. ${ }^{23,24}$

We first consider a case without electron-hole competition $\left(\xi_{0}= \pm 1\right)$. The imaginary part of the gain is here

$$
\gamma^{\prime \prime}=\frac{\pi n_{0}^{3} r_{\mathrm{eff}}}{\lambda \cos \theta} \frac{k_{B} T}{e} V \frac{k_{0}^{4}}{\left(k_{0}^{2}+k^{2}\right)^{2}+k^{2} V^{2}} .
$$

When the following conditions are verified:

(i) $k^{2} / k_{0}^{2} \ll 1$, i.e., a large grating spacing in front of the Debye screening length;

(ii) $\quad k^{2} V^{2} / k_{0}^{4} \ll 1$, i.e., a limited value of the applied electric field;

we obtain a large local effect, which is in proportion to the applied electric field $E_{0}$,

$$
\gamma^{\prime \prime}=\frac{\pi n_{0}^{3} r_{\mathrm{eff}} E_{0}}{\lambda \cos \theta}
$$

When $\xi_{0}=0$ and if conditions (i), (ii), and (iii) are verified:

(iii) $\bar{\kappa}^{2} / k_{0}^{2} \ll 1$, where $\bar{\kappa}^{2}=\left(\kappa_{n}^{2}+\kappa_{p}^{2}\right) / 2$ (i.e., for diffusion lengths longer than the Debye screening length that is usually verified in semiconductor),

we obtain

$$
\gamma^{\prime \prime}=\frac{\pi n_{0}^{3} r_{\mathrm{eff}} E_{0}}{\lambda \cos \theta} \frac{\bar{\kappa}^{4} k_{0}^{4}}{\left[k_{0}^{2} \bar{\kappa}^{2}+k^{2}\left(k_{0}^{2}+V^{2}\right)\right]^{2}} .
$$

This result is identical to the one obtained for $\xi_{0}= \pm 1$ Eq. (28) when a fourth condition is achieved,

(iv) $k^{2}\left(k_{0}^{2}+V^{2}\right) \ll k_{0}^{2} \vec{\kappa}^{2}$.

It, thus, appears that when the grating wave-number value is sufficiently low, the imaginary amplitude gain is equivalent in a crystal with or without electron-hole competition. This confirms our intuitive model.

In our CdZnTe:V crystals, we have, typically, $k_{0}$ $\approx 20 \mu \mathrm{m}^{-1}$, we apply an electric field smaller than $10 \mathrm{kV} \mathrm{cm}^{-1}$, which corresponds to $V<40 \mu \mathrm{m}^{-1}$ and, as usually admitted in the semiconductor, we consider that $\bar{\kappa}$ is smaller than or in the order of $1 \mu \mathrm{m}^{-1}$. We easily fulfill the first three conditions with $k$ in the order of $1 \mu \mathrm{m}^{-1}$, but the fourth one will be verified for much lower $k$ values only.

Consider now the general case $\left(-1 \leqslant \xi_{0} \leqslant 1\right)$. When the first three conditions are verified and for a $\mathrm{V}$ value, which is not negligible compared with $k$ (which means, for $k$ $=1 \mu \mathrm{m}^{-1}$, that the applied electric field is not negligible compared to $250 \mathrm{~V} \mathrm{~cm}^{-1}$ ), we obtain,

$$
\begin{aligned}
\gamma^{\prime \prime}= & \frac{\pi n_{0}^{3} r_{\mathrm{eff}} E_{0}}{\lambda \cos \theta} \\
& \times\left\{1-\frac{\left(1-\xi_{0}^{2}\right) k^{2}\left[k_{0}^{4}\left(k^{2}+\bar{\kappa}^{2}\right)+2 k_{0}^{2} V^{2} \bar{\kappa}^{2}+2 V^{4} k^{2}\right]}{k_{0}^{4}\left[\left(k^{2}+\bar{\kappa}^{2}-\xi_{0} \sigma_{\kappa^{2}}\right)^{2}+\xi_{0}^{2} V^{2} k^{2}\right]}\right\},
\end{aligned}
$$

where $\sigma_{\kappa^{2}}=\left(\kappa_{n}^{2}-\kappa_{p}^{2}\right) / 2$. The term, $\left(k^{2}+\bar{\kappa}^{2}-\xi_{0} \sigma_{\kappa^{2}}\right)^{2}$ $+\xi_{0}^{2} V^{2} k^{2}$, is the keypoint to understanding relation (30). We, thus, define a fifth condition:

(v) $\xi_{0}^{2} \gg\left(k^{2}+\bar{\kappa}^{2}-\xi_{0} \sigma_{\kappa^{2}}\right)^{2} / V^{2} k^{2}$.

When $(\mathrm{v})$ is verified, we obtain,

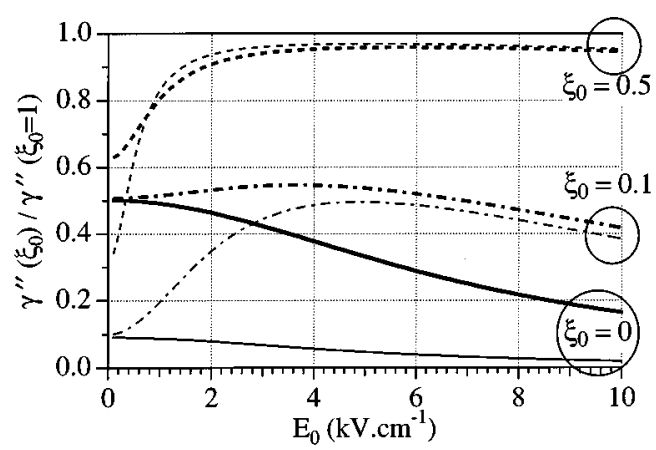

FIG. 2. $\gamma^{\prime \prime}\left(\xi_{0}\right) / \gamma^{\prime \prime}\left(\xi_{0}=1\right)$ as a function of the applied electric field for $k$ $=1 \mu \mathrm{m}^{-1}, k_{0}^{2}=384 \mu \mathrm{m}^{-2}$. The dashed curves correspond to $\xi_{0}=0.5$, the dashed-dotted ones to $\xi_{0}=0.1$, and the solid ones to $\xi_{0}=0$. The thick curves correspond to $\kappa_{n}^{2}=\kappa_{p}^{2}=1 \mu \mathrm{m}^{-2}$ and the thin ones to $\kappa_{n}^{2}=\kappa_{p}^{2}=0.1 \mu \mathrm{m}^{-2}$. The circles associate curves for a same $\xi_{0}$ value.

$$
\begin{aligned}
\left|\gamma^{\prime \prime}\right|= & \frac{\pi n_{0}^{3} r_{\mathrm{eff}} E_{0}}{\lambda \cos \theta}\left\{1-\frac{\left(1-\xi_{0}^{2}\right)}{\xi_{0}^{2}}\right. \\
& \left.\times\left(\frac{k^{2}+\vec{\kappa}^{2}}{V^{2}}+\frac{2 \vec{\kappa}^{2}}{k_{0}^{2}}+\frac{2 k^{2} V^{2}}{k_{0}^{4}}\right)\right\} .
\end{aligned}
$$

The last two terms are negligible, according to conditions (iii) and (ii), respectively, which gives

$$
\left|\gamma^{\prime \prime}\right|=\frac{\pi n_{0}^{3} r_{\mathrm{eff}} E_{0}}{\lambda \cos \theta}\left\{1-\frac{\left(1-\xi_{0}^{2}\right)\left(k^{2}+\bar{\kappa}^{2}\right)}{\xi_{0}^{2} V^{2}}\right\}
$$

the last term being negligible according to (v). So, when conditions (i-iii) and (v) are verified, the imaginary gain will verify relation (28) and will not depend on the electron-hole competition.

To illustrate this study, we present numerical simulations in Fig. 2 using relation (26). We represent the ratio $\gamma^{\prime \prime}\left(\xi_{0}\right) / \gamma^{\prime \prime}\left(\xi_{0}=1\right)$ as a function of the applied electric field using parameters of the B.V-4T3 sample given in Table I. The obtained results are very significant. There is a range of applied electric fields for which we observe that the electron-hole competition: (1) has nearly no effect for $\xi_{0}$ $=0.5$; (2) gives only a reduction of a factor 2 of $\gamma^{\prime \prime}$ when $\xi_{0}=0.1$; and (3) gives a reduction, which depends highly on the diffusing length when $\xi_{0}=0$.

This study uses a model with one deep level and two types of carriers. We showed that in some CdZnTe:V, it is necessary to take into account two types of deep level. ${ }^{25} \mathrm{We}$, thus, have conducted simulations using the model with two species $^{25,26}$ and have observed the same independence on the electron-hole competition. We can finally conclude that the electron-hole competition phenomenon is not detrimental to

TABLE I. Measured parameters in the B.V-4T3 sample.

\begin{tabular}{lcccc}
\hline \multicolumn{1}{c}{$\lambda$} & $1060 \mathrm{~nm}$ & $1320 \mathrm{~nm}$ & $1550 \mathrm{~nm}$ & $2000 \mathrm{~nm}$ \\
\hline$\alpha$ in $\mathrm{cm}^{-1}$ & 1.09 & 0.26 & 0.24 & 0.13 \\
$\xi_{0}$ & $0.57 \pm 0.03$ & $\approx 0.07$ & $-0.59 \pm 0.03$ & \\
$k_{0}^{2}$ in $\mu \mathrm{m}^{-2}$ & $384 \pm 35$ & & $336 \pm 35$ & \\
$N_{\text {eff }}$ in $\mathrm{cm}^{-3}$ & $(5.5 \pm 0.5) 10^{15}$ & & $(4.8 \pm 0.5) 10^{15}$ & \\
\hline \hline
\end{tabular}


the photorefractive sensor under a dc applied electric field, whereas it is greatly detrimental for similar systems working in the diffusion regime. We will now present measurements that confirm this theoretical analysis.

\section{EXPERIMENTAL STUDY OF THE PHOTOREFRACTIVE SENSOR SENSITIVITY}

We perform some experimental measurements of the sensitivity of our system as a function of the applied electric field. Our aim is to validate the insensitivity of the sensor to the electron-hole competition and to demonstrate the near optimal detection limit. We simulate the phase modulation of the signal beam using a Pockels cell. We first need to measure the phase shift introduced by this Pockels cell using a classical interferometric method. The accuracy of the following measurements depends on the one achieved in this calibration. We need a phase modulation much lower than $\pi / 2$ but sufficiently high to achieve measurements with high signal-to-noise ratios, even for a low applied electric field where the detection limit is very low. We experimentally obtain a root-mean-square phase modulation $\varphi_{\mathrm{rms}}=24 \pm 3 \mathrm{mrad}$, which corresponds to an equivalent displacement of $20 \mathrm{~nm} .24 \mathrm{mrad}$ is adapted to the first criterion $\left(\varphi_{\mathrm{rms}} \ll \pi / 2\right)$ and we obtain, with this phase modulation, high signal-to-noise ratios.

Then, we want to determine the real and the imaginary parts of the amplitude photorefractive gain as a function of the electric field applied to the crystal. The periodicity of the modulated signal introduced by the Pockels cell is chosen to be sufficiently short compared to the photorefractive response time. In this case, the output signal is given by Eq. (8).

The principle of the measurement is as follows: Without applying any phase modulation on the signal beam, we first measure the dc part of the detected signal in absence of the pump beam, $I_{0}=e^{-\alpha x} I_{s}(0,0)$, and in its presence, $\bar{I}$ $=e^{-\alpha x} e^{2 \gamma^{\prime} x} I_{s}(0,0)$. Then we measure, applying the phase modulation and always in the presence of the pump beam, the root-mean square of the detected signal, $\Delta I_{\mathrm{rms}}$ $=2 e^{-\alpha x} e^{\gamma^{\prime} x} I_{S}(0,0) \sin \left(\gamma^{\prime \prime} x\right) \varphi_{\mathrm{rms}}$. As we know $\varphi_{\mathrm{rms}}, I_{0}, \bar{I}$, $\Delta I_{\text {rms }}$ and $x$, we can calculate the $\gamma^{\prime},\left|\sin \left(\gamma^{\prime \prime} x\right)\right|$ and $\delta_{\text {rel }}$ values:

$$
\begin{aligned}
& \gamma^{\prime}=\ln \left(\bar{I} / I_{0}\right) / 2 x, \\
& \left|\sin \left(\gamma^{\prime \prime} x\right)\right|=\frac{1}{2} \frac{\Delta I_{\mathrm{rms}}}{\sqrt{\bar{I} \cdot I_{0}} \frac{1}{\varphi_{\mathrm{rms}}},} \\
& \delta_{\mathrm{rel}}=\frac{e^{\alpha x / 2}}{\left|\sin \left(\gamma^{\prime \prime} x\right)\right|}=2 e^{\alpha x / 2} \frac{\sqrt{\bar{I} \cdot I_{0}}}{\Delta I_{\mathrm{rms}}} \varphi_{\mathrm{rms}} .
\end{aligned}
$$

We now apply this principle to the measurement of the photorefractive gain as a function of the applied electric field in two of our best CdZnTe samples. We here use three laser sources: one emitting at $\lambda=1.06 \mu \mathrm{m}$ because many highpower lasers have already been developed at this wavelength, another emitting at $\lambda=1.55 \mu \mathrm{m}$ because it could become an alternative to $1.06 \mu \mathrm{m}$ thanks to its eye-safe properties, ${ }^{27}$ and the last one emitting at $\lambda=1.32 \mu \mathrm{m}$ because
TABLE II. Measured parameters in the B.V-4T6 sample.

\begin{tabular}{ccccc}
\hline \hline \multicolumn{1}{c}{$1060 \mathrm{~nm}$} & $1320 \mathrm{~nm}$ & $1550 \mathrm{~nm}$ & $2000 \mathrm{~nm}$ \\
\hline$\alpha$ in $\mathrm{cm}^{-1}$ & 1.57 & 0.41 & 0.30 & 0.08 \\
$\xi_{0}$ & $0.62 \pm 0.03$ & $\approx 0.1$ & $-0.41 \pm 0.03$ & \\
$k_{0}^{2}$ in $\mu \mathrm{m}^{-2}$ & $375 \pm 35$ & & $206 \pm 35$ & \\
$N_{\text {eff }}$ in $\mathrm{cm}^{-3}$ & $(5.4 \pm 0.5) 10^{15}$ & & $(3 \pm 0.5) 10^{15}$ & \\
\hline \hline
\end{tabular}

there is, at this wavelength, a large electron-hole competition in our samples, which will allow us to validate the insensitivity of our photorefractive sensor to this competition.

The samples tested are called B.V-4T3 $\left(\mathrm{Cd}_{0.96} \mathrm{Zn}_{0.04} \mathrm{Te}: \mathrm{V}\right)$ and B.V-4T $6(\mathrm{CdTe}: \mathrm{Zn}: \mathrm{V})$. They are large-sized single crystals oriented in a classical geometry: two polished (110) faces, two (110) faces, and two (001) faces. Note that these two samples are, respectively, 2 and 1 $\mathrm{cm}$ thick. At a wavelength of $2 \mu \mathrm{m}$, where absorption due to the vanadium defect is negligible, we have almost no absorption (typically, only $0.1 \mathrm{~cm}^{-1}$ ), contrary to the sample we had previously used. 15,28

A measurement of the photorefractive gain as a function of the grating wave number, $k(k=2 \pi / \Lambda$, where $\Lambda$ is the grating spacing), allows us to determine two essential photorefractive parameters: the absolute value of the electron-hole competition factor, $\xi_{0}$, and the effective trap density, $N_{\text {eff }}$ $=\left(\epsilon k_{B} T / e^{2}\right) k_{0}^{2}\left(\epsilon\right.$ is the dielectric constant of the material, $k_{B} T$ is the thermal energy, $e$ is the modulus of the electron charge, and $k_{0}$ is the inverse of the Debye screening length), of the sample. ${ }^{23,24}$ We make copropagating measurements, i.e., using transmission grating, and counterpropagating measurements, i.e., using reflection grating, to determine these two photorefractive parameters with a good accuracy. ${ }^{25}$ In parallel, we also determine the sign of the majority carriers by measuring both the sign of the photorefractive gain and of the effective electro-optic coefficient. ${ }^{28}$ We obtain similar results in the two tested crystals (Tables I and II) with electrons as dominating carriers at $1.06 \mu \mathrm{m}$, near perfect electron-hole competition at $1.32 \mu \mathrm{m}$, and holes at $1.55 \mu \mathrm{m}$.

Experiments under an applied voltage have been made for an angle of the order of $10^{\circ}$ between the two beams outside the crystal, corresponding at $\lambda=1.06 \mu \mathrm{m}$ to a grating spacing of $\Lambda=6.4 \pm 0.4 \mu \mathrm{m}\left(k=1 \mu \mathrm{m}^{-1}\right)$. We here work with a grating wave vector along the [110] direction and with beams polarized at $45^{\circ}$ of the [001] and [110] directions. In this configuration, we obtain $r_{\text {eff }}=r_{41}$, and we can easily go from an amplification regime to an attenuation one rotating the polarization by $90^{\circ} .{ }^{29}$ Measurements at $\lambda=1.06 \mu \mathrm{m}$ have been made under the following conditions:

(1) The irradiance of the pump beam in the crystal is $32 \mathrm{~mW} \mathrm{~cm}^{-2}$. It is greatly sufficient to neglect the influence of the dark conductivity in comparison to the photoconductivity.

(2) The pump-to-signal irradiance ratio is 64 , which is sufficiently high to neglect the depletion of the pump beam.

(3) The crystal is uniformly illuminated by the pump beam. This is an essential precaution to limit some critical screening effect of the electric field. 
(4) Inside the crystal, the signal beam has a small diameter, which guarantees that it is fully covered by the pump beam in the whole crystal.

(5) Finally, to prevent a potential critical heating of the crystal during the dc electric-field application, we only apply it during a short time, typically, $50 \mathrm{~ms}$, which is sufficient to create the hologram in the crystal and to measure ultrasonic vibrations. Moreover, we control the conductivity of the crystal during this field application. In fact, we have tested the system with a dc field application up to $3 \mathrm{~s}$ without any slightest problem.

First measurements were conducted in the B.V-4T3 at $1.06 \mu \mathrm{m}$. We measure $\gamma^{\prime}$ and $\left|\sin \left(\gamma^{\prime \prime} x\right)\right|$ as a function of the applied electric field in both the amplification and attenuation regimes. We observe a difference between the real part of the photorefractive amplitude gain in the attenuation and in the amplification regime (Fig. 3) that we currently do not explain. We determine that $\left|\sin \left(\gamma^{\prime \prime} x\right)\right|$ equals 1 for an applied electric field of the order of $4 \mathrm{kV} \mathrm{cm}^{-1}$ (Fig. 4). We then obtain a relative detection limit [see Eq. (10)] of $e^{\alpha x / 2}=3$, which is quite good. We also notice that after having achieve the value of $1,\left|\sin \left(\gamma^{\prime \prime} x\right)\right|$ stays almost constant, when we increase the applied electric field.

The parameter used for the theoretical curves are those presented in Table I $\left(N_{\text {eff }}, \xi_{0}\right)$. To adjust theoretical curves with the measurements, we introduce a screening effect factor. ${ }^{30}$ Applying to the crystal a voltage $U$, the interelectrode distance being $d$, we note the effective field applied to the crystal $p U / d . p$ takes into account edge effects and the Gaussian distribution of the pump beam. The optimal adjustment between theory and experiments is obtained for $p$ $=0.5$ (Figs. 3 and 4).

The diffusion length of electrons $\left(1 / \kappa_{n}\right)$ and holes $\left(1 / \kappa_{p}\right)$ are indeterminate parameters. In fact, the most influent parameter is $\bar{\kappa}$. We plot, in Figs. 3 and 4 , the curves obtained for different $\kappa_{n}=\kappa_{p}=\bar{\kappa}$ values. We observe that the curves obtained for $\kappa_{n}^{2}, \kappa_{p}^{2} \ll k^{2}$ are well adjusted with the experiments for $U / d<5 \mathrm{kV} \mathrm{cm}^{-1}$. For higher voltages, we were theoretically expecting a slower increase of $\gamma^{\prime}$ and a more important reduction of $\sin \left(\gamma^{\prime \prime} x\right)$. The experimental behavior at high voltages is not well understood for the moment.

The same experiments made at $1.55 \mu \mathrm{m}$ give us $\left|\sin \left(\gamma^{\prime \prime} x\right)\right|$ of 0.8 for an applied electric field of about $6 \mathrm{kV} \mathrm{cm}^{-1}$. This corresponds to a relative detection limit of 1.6 , which is very close to the ideal case.

We now present measurements made with the B.V-4T6 crystal, which has a thickness of only $1 \mathrm{~cm}$ instead of $2 \mathrm{~cm}$ for B.V-4T3. Figure 5 presents $\left|\sin \left(\gamma^{\prime \prime} x\right)\right|$ as a function of $U / d$ for the three wavelengths. At $1.06 \mu \mathrm{m},\left|\sin \left(\gamma^{\prime \prime} x\right)\right|$ reaches 1 for $U / d=9 \mathrm{kV} \mathrm{cm}^{-1}$, thus, giving a relative detection limit of $\delta_{\text {rel }}=2.2$. At $1.55 \mu \mathrm{m}$, the maximum applied voltage is not sufficient to reach 1 , but we nevertheless obtain $\left|\sin \left(\gamma^{\prime \prime} x\right)\right|=0.6$, and then $\delta_{\text {rel }}=1.9$. Finally, at $1.32 \mu \mathrm{m}$, we observe a saturation of $\left|\sin \left(\gamma^{\prime} x\right)\right|$ for $U / d=6 \mathrm{kV} \mathrm{cm}^{-1}$ with an optimal value of 0.65 and then $\delta_{\text {rel }}=1.9$. We note that the electron-hole competition factor at this wavelength is $\xi_{0} \cong 0.1$. This validates the rather good insensitivity of the technique to electron-hole competition. Whatever the wavelength, we now nearly reach the detection sensitivity of a classical homodyne interferometer.

Remembering that the photorefractive gain is inversely proportional to $\lambda$, we plot, in Fig. $6, \lambda \gamma^{\prime \prime}$ for each of the three wavelengths as a function of $U / d$. Three nearly superposed curves are obtained for $U / d \leqslant 6 \mathrm{kV} \mathrm{cm}^{-1}$, even if the points obtained at $1.06 \mu \mathrm{m}$ are slightly above the other ones. This difference increases when $U / d$ exceeds $6 \mathrm{kV} \mathrm{cm}^{-1}$. We can, therefore, reasonably conclude that the imaginary gain, corrected from wavelength scaling variations, has a similar amplitude despite significant variations of the electron-hole competition factor from one wavelength to another $(0.6,0.1$, and -0.6 at $\lambda=1.06,1.32$, and $1.55 \mu \mathrm{m}$, respectively).

\section{STUDY OF THE FREQUENCY RESPONSE OF THE SENSOR}

We here want to determine the low cutoff frequency of our setup and eventually find a configuration that gives the highest cutoff frequency value for a fixed pump beam irradiance. The large thickness of the B.V-4T3-B.V-4T6 samples allows us a more accurate study of the frequency response than previously with sample B23. ${ }^{15}$ With this B23 sample, we observed a slight resonance phenomenon, an overshoot that appears for a periodicity of the phase modulation in the order of the response time of the photorefractive effect. We are here expecting a higher overshoot, due to the increase of sample thickness.

We begin this analysis by a first observation. We measure the buildup of the photorefractive energy transfer gain $\left(\Gamma=2 \gamma^{\prime}\right)$ when the applied electric field goes abruptly from 0 to $10 \mathrm{kV} \mathrm{cm}^{-1}$. This measurement is made in both amplification and attenuation configurations (Fig. 7). These curves show that the buildup of the photorefractive gain is faster in the attenuation regime than in the amplification one by an order of magnitude. This result leads us to compare the frequency response of our homodyne detector working either in the attenuation or in the amplification regime. It is expected to obtain a higher low cutoff frequency for the sensor operating in the attenuation regime (attenuation and amplification regimes are identical for the detection sensitivity as long as the detected signal is photon noise limited).

We measure the frequency response with the B.V-4T3 sample at $\lambda=1.06 \mu \mathrm{m}$ under a $U / d=4 \mathrm{kV} \mathrm{cm}^{-1}$ electric field. The pump beam irradiance is $22 \mathrm{~mW} \mathrm{~cm}^{-2}$. The sensitivity of the system at frequency $f$ is normalized to the one obtained for rapidly phase modulated signals. It clearly appears that the overshoot only exists in the amplification gain regime (Fig. 8). We observe in this regime a large overshoot at $60 \mathrm{~Hz}$, which is 2.4 times higher than the response at rapid modulation frequency.

We define the cutoff frequency, $f_{c}$, as the phase modulated frequency under which the normalized response is less than 0.5 . We then obtain a cutoff frequency about four times higher in the attenuation regime $\left(f_{c} \approx 40 \mathrm{~Hz}\right)$ than in the amplification one $\left(f_{c} \approx 10 \mathrm{~Hz}\right)$ at $22 \mathrm{~mW} \mathrm{~cm}^{-2}$ pump beam irradiance.

This phenomenon is perfectly described theoretically. For this, we here use expressions (1) and (3) to calculate the 


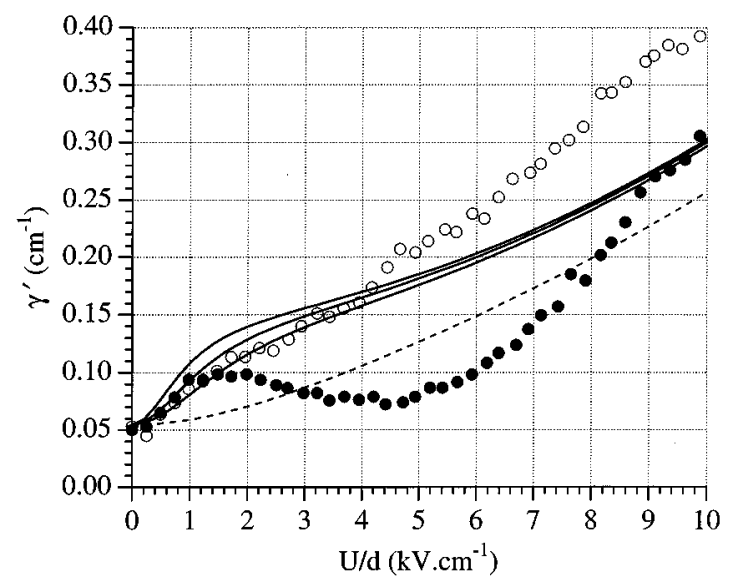

FIG. 3. Measurement of $\gamma^{\prime}$ as a function of the applied electric field with the B.V-4T3 sample at $1.06 \mu \mathrm{m}$. The empty and full circles correspond to an experimental measurement in the amplification and attenuation regime, respectively. Three solid theoretical curves are represented. They correspond to $\kappa_{n}^{2}=\kappa_{p}^{2}=0.1,0.5$, or $1 \mu \mathrm{m}^{-2}$. The dashed theoretical curve corresponds to $\kappa_{n}^{2}=\kappa_{p}^{2}=5 \mu \mathrm{m}^{-2}$.

frequency response in the two regimes. In the drift regime, the photorefractive response time at the entrance of the crystal $(x=0), \tau_{0}$, verifies relation ${ }^{22}$

$\tau_{0}=\frac{\epsilon k_{B} T / I_{0} e^{2}}{\left[\frac{A_{n} \alpha_{n}}{\kappa_{n}^{2}}\left(\frac{k(k-i V) / k_{0}^{2}+1}{k(k-i V) / \kappa_{n}^{2}+1}\right)+\frac{A_{p} \alpha_{p}}{\kappa_{p}^{2}}\left(\frac{k(k+i V) / k_{0}^{2}+1}{k(k+i V) / \kappa_{p}^{2}+1}\right)\right]}$,

where $\epsilon$ is the dielectric constant of the material, and $I_{0}$ is the irradiance at the entrance of the crystal. So, $\tau_{0}$ is a complex number characterized by its modulus, $\left|\tau_{0}\right|$, and by its phase, $\varphi_{\tau_{0}}$.

In Eqs. (1) and (3), we make the $u=t /\left|\tau_{0}\right|$ variable change, so that the $\left|\tau_{0}\right|$ parameter disappears. We also normalize the frequency response: the only effect of a change of

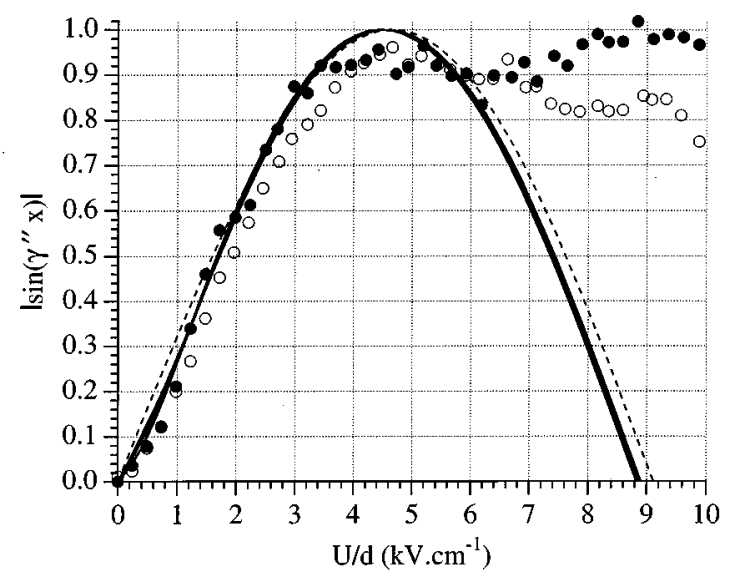

FIG. 4. Measurement of $\left|\sin \left(\gamma^{\prime \prime} x\right)\right|$ as a function of the applied electric field with the B.V-4T3 sample at $1.06 \mu \mathrm{m}$. The empty and full circles correspond to an experimental measurement in the amplification and attenuation regimes, respectively. Theoretical curves, calculated for the same parameters as the one in Fig. 3, are presented. It appears that the three solid curves are superposed.

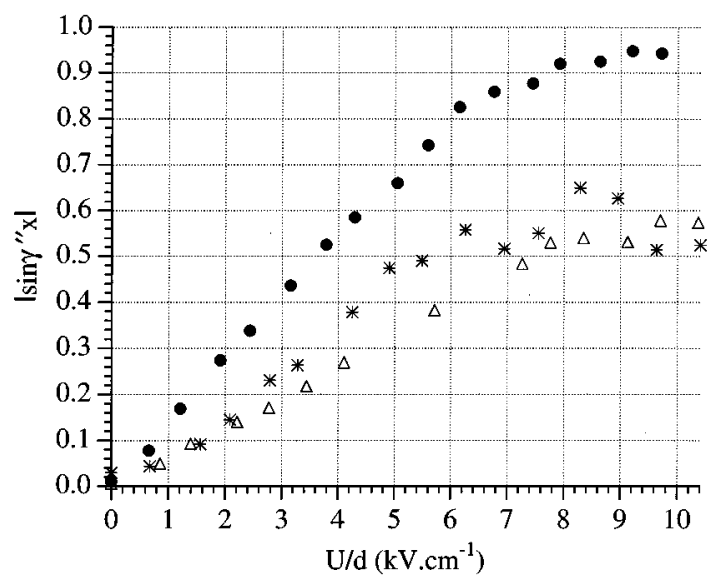

FIG. 5. Measurement of $\left|\sin \left(\gamma^{\prime \prime} x\right)\right|$ as a function of $U / d$ with the B.V-4T6 at $1.06 \mu \mathrm{m}$ (full circles), $1.32 \mu \mathrm{m}$ (stars), and $1.55 \mu \mathrm{m}$ (empty triangles).

$\left|\tau_{0}\right|$ is a dilatation on the frequency axis, which can be easily obtained by changing illumination $I_{0}$. At a field $U / d$ $=4 \mathrm{kV} \mathrm{cm}^{-1}$ of the measurement, we know gain $\gamma$, absorption $\alpha$, and thickness $x$ of the B.V-4T3 crystal (Table I). These parameters are used for the calculation of the frequency response in both amplification and attenuation regimes for different values of $\varphi_{\tau_{0}}$ (Fig. 8). It theoretically validates the presence of a high overshoot in the amplification regime and of none in the attenuation one (except a small overshoot for $\varphi_{\tau_{0}}=0$ ). We observe, in the amplification regime, that the size of the overshoot is a rising function of $\varphi_{\tau_{0}}$ and that the low cutoff frequency is a decreasing function of $\varphi_{\tau_{0}}$. We observe an opposite effect in the attenuation regime where the low cutoff frequency increases with $\varphi_{\tau_{0}}$. We obtain a good fit between theory and experiments for $\left|\tau_{0}\right|=1.1 \mathrm{~ms}$ and for a phase of the response time of 0.7 $\operatorname{rad}$ (Fig. 8).

\section{CONCLUSION}

We have theoretically and experimentally studied a photorefractive beam combiner for optical detection of ultra-

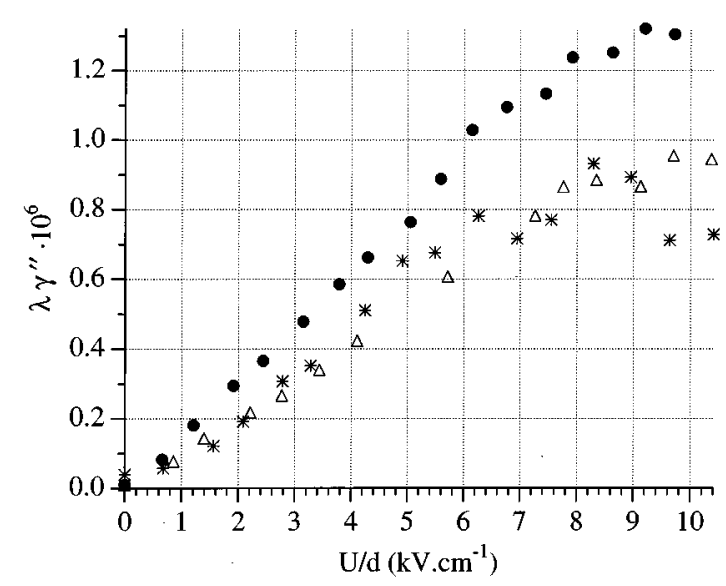

FIG. 6. Plots of $\lambda \gamma^{\prime \prime}$ as a function of $U / d$, deduced from measurements presented in Fig. 5, with the B.V-4T6 at $1.06 \mu \mathrm{m}$ (full circles), $1.32 \mu \mathrm{m}$ (stars), and $1.55 \mu \mathrm{m}$ (empty triangles). 

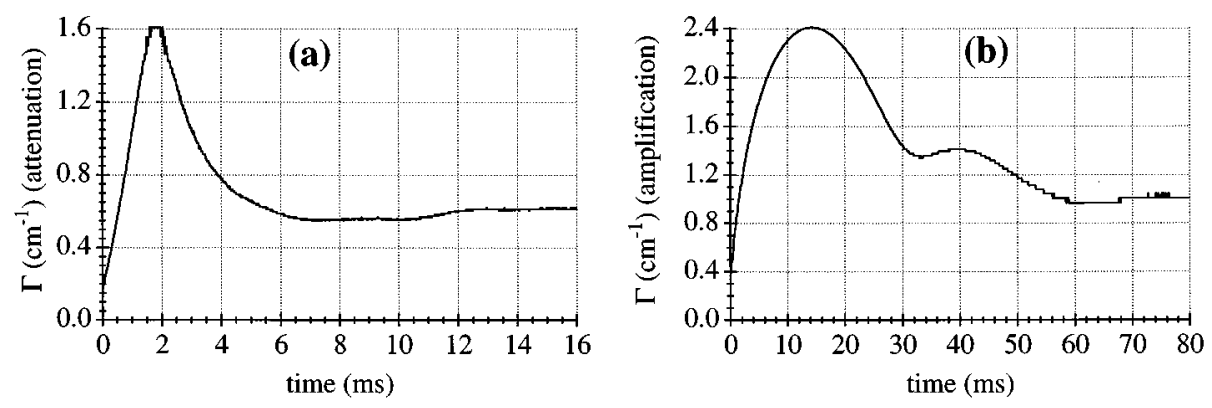

FIG. 7. Experimental buildup of the two-wave-mixing photorefractive energy transfer gain, $\Gamma$, under applied electric field in the attenuation regime (a) and in the amplification one (b).

sound waves on rough surfaces. We show that there is a good agreement between theory and experiments for both photorefractive effects (characterizations) and phase modulation detection (sensitivity and frequency response to ultrasonic displacements). We also demonstrate that in our configuration, a response, independent of the electron-hole competition in the photorefractive crystal, is obtained, leading to a system that can be developed at different wavelengths with identical performances and the same crystal. With the development of large size $\left(>1 \mathrm{~cm}^{3}\right)$ and optimized crystals, we have now a system, working with speckled beams, that is almost as sensitive as a classical heterodyne interferometer working with plane waves. It is more sensitive than the confocal Fabry-Perot interferometer on a large flat frequency bandwidth. The high pass frequency response of the system is confirmed with the observation, for a given crystal orientation and beams polarizations, of an overshoot. The low cutoff frequency achieved in cw experiments $(\approx 40 \mathrm{~Hz})$ was linked to the low-power laser we used and can be increased $(1-10 \mathrm{kHz})$ to eliminate the ambient vibrations by an increase of the power of the laser (for a pump beam irradiance going, typically, from 1 to $10 \mathrm{~W} \mathrm{~cm}^{-2}$ ). Measurements per-

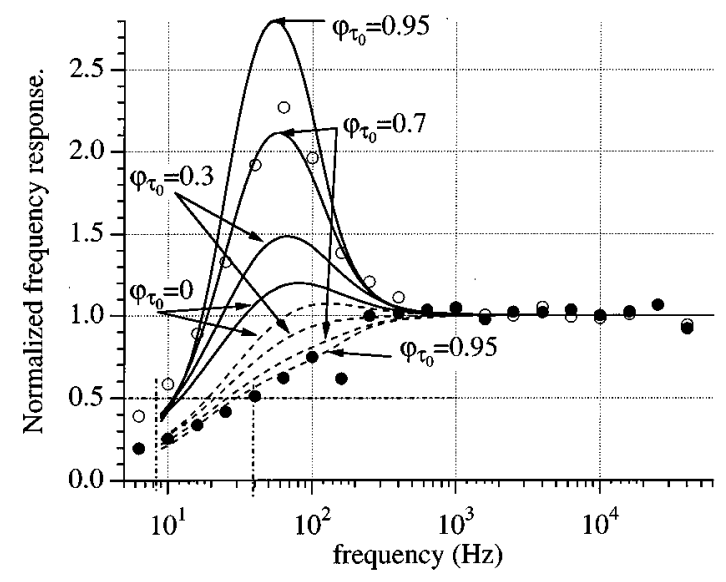

FIG. 8. Experimental frequency response of the system under a dc field application of $4 \mathrm{kV} \mathrm{cm}^{-1}$. Theoretical curves are obtained, adjusting the $\left|\tau_{0}\right|$ value only, and for a phase of the response time of $0,0.3,0.7$, and $0.95 \mathrm{rad}$. The height of the overshoot increases with the phase of the response time in the amplification regime. The low cutoff frequency decreases with the phase of the response time in the amplification regime and increases in the attenuation one. The solid line curves correspond to the theory in the amplification regime and the dotted ones to the theory in the attenuation regime. formed with a high-power pulsed laser on a InP crystal ${ }^{16,17}$ and on one of the CdZnTe crystals used in this study confirm that such cutoff frequencies can be obtained without loss in detection sensitivity. Considering the results of this analysis, optimization of CdZnTe will be continued with the view to obtain new crystals that exhibit the same performances in more relaxed conditions (smaller applied field, smaller pump beam power, ...). We will also assess the reproducibility of the crystal growth. An industrialization of the system will be done in parallel on the basis of the laboratory prototype developed in the course of this study.

${ }^{1}$ C. B. Scruby and L. E. Drain, Laser Ultrasonics: Techniques and Applications (Hilger, Bristol, United Kingdom, 1990).

${ }^{2}$ J.-P. Monchalin, IEEE Trans. Ultrason. Ferroelectr. Freq. Control 33, 485 (1986).

${ }^{3}$ E. S. Boltz, C. M. Fortunko, M. A. Hamstad, and M. C. Renken, Rev. Prog. Quant. Nondestruct. Eval. 14, 967 (1995); E. S. Boltz and C. M. Fortunko, ibid. 15, 939 (1996).

${ }^{4}$ J.-P. Monchalin and R. Héon, Mater. Eval. 44, 1231 (1986).

${ }^{5}$ J.-P. Monchalin, R. Héon, P. Bouchard, and C. Padioleau, Appl. Phys. Lett. 55, 1612 (1989).

${ }^{6}$ LISOR, Optical Detection of Ultrasound, UltraOptec Inc., 27, rue de Lauzon, Boucherville, (Québec,) J4B 1E7 Canada.

${ }^{7}$ L. E. Adams and R. S. Bondurant, Opt. Lett. 16, 832 (1991).

${ }^{8}$ H. Nakano, Y. Matsuda, S. Shin, and S. Nagai, Ultrasonics 33, 261 (1995).

${ }^{9} \mathrm{Ph}$. Delaye, A. Blouin, D. Drolet, and J.-P. Monchalin, Appl. Phys. Lett. 67, 3251 (1995).

${ }^{10}$ N. Korneev, P. Rodriguez, B. Sanchez, and S. Stepanov, Optik (Stuttgart) 102, 21 (1996).

${ }^{11}$ Y. O. Barmenkov, V. V. Zosimov, N. M. Kozhevnikov, L. M. Lyamshev, and S. A. Sergushschenko, Sov. Phys. Dokl. 31, 817 (1986).

${ }^{12}$ R. K. Ing and J.-P. Monchalin, Appl. Phys. Lett. 59, 3234 (1991).

${ }^{13}$ A. Blouin and J.-P. Monchalin, Appl. Phys. Lett. 65, 932 (1994).

${ }^{14}$ T. Honda, T. Yamashita, and H. Matsumoto, Jpn. J. Appl. Phys., Part 1 34, 3737 (1995)

${ }^{15}$ L.-A. de Montmorillon, I. Biaggio, Ph. Delaye, J.-C. Launay, and G. Roosen, Opt. Commun. 129, 293 (1996).

${ }^{16} \mathrm{Ph}$. Delaye, A. Blouin, L.-A. de Montmorillon, I. Biaggio, D. Drolet, J.-P. Monchalin, and G. Roosen, Proc. SPIE 2782, 50 (1996).

${ }^{17} \mathrm{Ph}$. Delaye, A. Blouin, D. Drolet, L.-A. de Montmorillon, G. Roosen, and J.-P. Monchalin, J. Opt. Soc. Am. B 14, 1723 (1997).

${ }^{18} \mathrm{Ph}$. Delaye, L.-A. de Montmorillon, and G. Roosen, Opt. Commun. 118, 154 (1995).

${ }^{19}$ M. Abramowitz and I. A. Stegun, Handbook of Mathematical Functions, 1st ed. (Dover, New York, 1965).

${ }^{20}$ C. T. Field and F. M. Davidson, Appl. Opt. 32, 5285 (1993).

${ }^{21}$ M. P. Petrov, S. I. Stepanov, and A. V. Khomenko, Photorefractive Crystals in Coherent Optical Systems (Springer-Verlag, Berlin, 1991).

${ }^{22}$ F. P. Strohkend1, J. M. C. Jonathan, and R. W. Hellwarth, Opt. Lett. 11, 312 (1986).

${ }^{23}$ M. B. Klein and G. C. Valley, J. Appl. Phys. 57, 4901 (1985). 
${ }^{24}$ L.-A. de Montmorillon, Ph. Delaye, J.-C. Launay, and G. Roosen, Opt. Mater. 4, 233 (1995).

${ }^{25} \mathrm{Ph}$. Delaye, L.-A. de Montmorillon, I. Biaggio, J.-C. Launay, and G. Roosen, Opt. Commun. 134, 580 (1997).

${ }^{26}$ M. C. Bashaw, M. Jeganathan, and L. Hesselink, J. Opt. Soc. Am. B 11, 1743 (1994).

${ }^{27}$ P. S. Avdeev, Y. D. Berezin, Y. P. Gudakovskii, V. R. Muratov, A. G.
Murzin, and V. A. Fromzel, Sov. J. Quantum Electron. 81, 137 (1978).

${ }^{28}$ L.-A. de Montmorillon, thése de doctorat No. 4776, Université Paris XI Orsay (1997).

${ }^{29}$ J. C. Fabre, J. M. C. Jonathan, and G. Roosen, Opt. Commun. 65, 257 (1988).

${ }^{30}$ J.-M. C. Jonathan, R. W. Hellwarth, and G. Roosen, IEEE J. Quantum Electron. QE-22, 1936 (1986). 